INT. J. GEOGRAPHICAL INFORMATION SYSTEMS, 1996, VOL. 10, NO. 5, 605-627

Research Article

\title{
The geography of parameter space: an investigation of spatial non-stationarity
}

\author{
A. STEWART FOTHERINGHAM, MARTIN CHARLTON
}

NE.RRL/Department of Geography, University of Newcastle upon Tyne, Newcastle upon Tyne, NE1 7RU, England, U.K.

\section{and CHRIS BRUNSDON}

NE.RRL/Department of Town and Country Planning, University of Newcastle upon Tyne, Newcastle upon Tyne, NE1 7RU, England, U.K.

(Received 27 September 1994; Accepted 3 March 1995)

\section{Introduction}

In line with the current information revolution, geographers increasingly make a distinction between the analysis of spatial data and spatial data analysis. While both types of analysis investigate data having geographical co-ordinates, the former effectively ignores the geographical component and treats the data as if they were aspatial (inter alia, Flowerdew and Green 1994), whereas the latter makes use of the geographical component to explore explicitly spatial aspects of the data (inter alia, Anselin 1988, Besag 1986). It is this latter characteristic which makes spatial data analysis such a rich field for exploratory investigation. To clarify the distinction, suppose one is interested in the relation between an attribute $Y$ and a set of attributes $X_{1}, X_{2} \ldots X_{N}$ where the data all have spatial co-ordinates. A frequently used procedure to examine relations in such a situation is to regress $Y$ on the set of $X$ variables using the full data set. That is, observations from all the spatial units are used to produce a 'global' set of parameter estimates that relate the variable $Y$ to each of the $X$ 's. In following such a procedure, a great deal of spatial information is lost in that the relations being examined may in fact exhibit significant spatial variation which is not uncovered in the estimation of the global parameter estimates. This variation is referred to as spatial non-stationarity.

In this paper, we propose an exploratory method of spatial data analysis which allows spatial variations in relations to be examined visually. It is useful to note at this stage that exploratory analysis is of two types: pre-confirmatory, which focusses on data accuracy, and post-confirmatory which focusses on model accuracy. However, neither type of exploratory analysis is exclusively data or model-orientated. Pre-confirmatory exploratory analysis can be used to suggest relations to be included within a modelling framework and post-confirmatory analysis can be useful in highlighting unusual aspects of data. The exploratory analysis described here is postconfirmatory and we are concerned with examining differences between a 'global' calibration of a model and many different 'local' calibrations. In particular, we are interested in exploring spatial variations in model calibration results. We would argue that this method has the following advantages over more conventional analysis: 
1. It allows greater insights into the nature and accuracy of the data being examined;

2. It provides a more detailed understanding of the nature of relations and their variation over space;

3. It demonstrates the possible naiveté of conventional approaches to data analysis that often ignore spatial non-stationarity; and

4. It allows a more detailed comparison of the relative performances of different types of analysis or different models.

\section{Visualisation of spatial non-stationarity}

Consider a region divided into $\mathbf{R}$ rows and $\mathbf{C}$ columns in which a typical analysis is undertaken so that $\mathbf{R}$ by $\mathbf{C}$ observations are used to produce a single set of parameter estimates (the analysis of spatial data or global approach). An alternative technique involves placing a moving window of dimensions $\mathbf{r}$ by $\mathbf{c}$ (where $\mathbf{r}<\mathbf{R}$ and $\mathbf{c}<\mathbf{C})$ over every cell of the matrix for which the window can be centred without any part of it lying outside the region. For every placement of the moving window the relation between $Y$ and each of the $X$ 's is estimated so that a set of parameter estimates for each relation is obtained (see figure 1). These sets of parameter estimates have spatial co-ordinates and can therefore be mapped to show how the relation between $Y$ and a particular $X$ varies over space (a spatial data analysis or local approach). The distinction between the terms global and local as used here is similar to that between the analysis of spatial data and spatial data analysis. Obviously a spatial goodness-of-fit statistic and other diagnostics can be mapped in the same way. The key outputs then are two- and three-dimensional surfaces of parameter estimates, their $t$-values and an $R$-square statistic. Two-dimensional plots are also presented to compare the relative performance of global and local calibration methods.

It is noted at the outset that the detection of spatial non-stationarity depends on a subjective impression of the roughness of a three-dimensional surface of parameter estimates although work is currently in progress to produce a more objective comparative procedure. Clearly, if spatial non-stationarity does not exist, the parameter

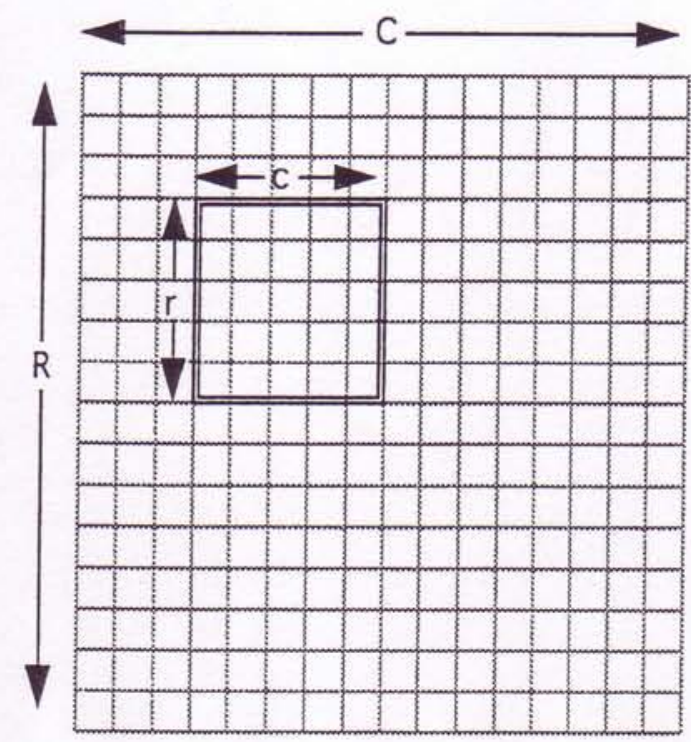

Figure 1. Moving window parameter estimation. 
surface depicting spatial variations in relations will be smooth and increasing spatial non-stationarity will be indicated by increasing divergence from this smooth surface.

It is further noted that a square window is being employed and that other shapes of windows could be used. Perhaps the most intuitive window shape would be circular so that cells are included that are within a pre-specified distance from the centre of the window. However, a square window was chosen as being more representative of regions that are often defined for data analysis. It is more likely, for example, that a square or rectangular subset of the data is used to calibrate a model-rarely are study regions circular. As an example, the global region which forms the basis of this analysis and which is described below, is rectangular.

The technique proposed is similar to other developments in spatial data analysis such as those related to the modifiable areal unit problem by, inter alia, Fotheringham and Wong (1991) where the sensitivity of analytical results to variations in the spatial units for which data are observed is examined. Both studies utilise GIS to examine spatial aspects of the sensitivity of model performance. However, whereas the investigations of the modifiable areal unit problem keep the study area boundary fixed and examine the performance of an analytical method when the study space is divided in different ways, the technique described here in effect varies the study region so that different subsets of the data are used to calibrate a model. The technique is also part of a growing field of analytical techniques that provide spatial disaggregations of more traditional global statistics. Other examples include the development of a localised spatial association statistic by Getis and Ord (1991), the generation of variogram clouds and pocket plots by Cressie (1991), and the spatial expansion of parameter estimates by Jones and Casetti (1992) and by Fotheringham and Pitts (1995).

\section{An example using OLS and bi-square regression}

The basic aim of this example is to examine the presence of spatial nonstationarity in the calibration of a regression model. The model attempts to connect people and the environment by investigating the relations between population density and a series of attributes of the physical landscape, namely: elevation, land cover and soil type. The data on these variables are all taken from an area in north-east Scotland and are described more fully below.

\subsection{Data}

The data refer to an area $38.45 \mathrm{~km}$ by $51.2 \mathrm{~km}$ in the north-east of Scotland which is described in detail in Aspinall and Veitch (1993) and consist of a population density measure as the dependent variable, and ground elevation, land cover, and soil type as the independent variables. The object of this example is to provide a demonstration of the visualisation of spatial non-stationarity rather than to model the socio-physical processes affecting the settlement pattern in the study area, but in the interests of having a reasonably plausible model on which to work, it might be expected that the distribution of population is related to land cover, elevation, and (perhaps more speculatively) the underlying soil type. The models are based on a $1 \mathrm{~km}$ raster.

Counts of residential population for each census enumeration district in the study area were extracted from the 1991 Census of Population together with the co-ordinates of the centroid of each enumeration district. The count data were aggregated to the $1 \mathrm{~km}$ raster on an 'all or nothing' basis - the raster is relatively 
crude by comparison with that used by Martin (1989) in allocating centroid based census data to a raster. The density for a given grid square $i$ is calculated as:

$$
\text { POPDEN }_{i}=\sum_{j}\left(\text { population }_{j} / \sqrt{ }\left(\left(x_{j}-x_{i}\right)^{2}+\left(y_{j}-y_{i}\right)^{2}\right)\right)
$$

where $\left(x_{j}, y_{j}\right)$ is the co-ordinate of the lower left-hand co-ordinate of grid square $j$.

The elevation raster was resampled from a 1:250000 scale digital terrain model, originally sampled every $100 \mathrm{~m}$. The land cover data were extracted from a $50 \mathrm{~m}$ raster coverage, captured at 1:50000-the data were reclassed into 'woodland', 'grassland' and 'other', each being expressed as a percentage of the land area in a $1 \mathrm{~km}$ output raster. The soils data were extracted from $100 \mathrm{~m}$ raster data, captured at 1:250000 scale and reclassed into 'podsols' and 'other soil types'; in the output raster, the percentage of the land area that is composed of podsols is recorded.

\subsection{OLS regression}

The above data were standardised by transforming them into Z-scores and these were used to calibrate the following model:

$$
P O P D E N=\alpha+\beta_{1} E L+\beta_{2} W O O D+\beta_{3} G R A S S+\beta_{4} P O D S
$$

such that global estimates of the four slope parameters as well as a global goodnessof-fit indicator, the $R$-squared statistic, were obtained. The globally fitted model is:

$$
P O P D E N=0-0.43 \text { EL }-0.05 \text { WOOD }-0.11 \text { GRASS +0.10 PODS }
$$

with an $R$-squared value of $0 \cdot 26$. The values in parentheses are the absolute values of the $t$-statistics associated with each parameter estimate.

The results suggest that although there remains a considerable amount of variance to be explained, there do seem to be some significant relations between population density and features of the physical landscape. Population density decreases significantly as elevation rises, as the proportion of both woodland and grassland rises, and increases significantly in areas where podsols are found. A not unreasonable interpretation of these results would be that in this part of Scotland, settlements are found primarily in low-lying areas which are cleared of woodland and grassland and which have fairly rich podsolic soils conducive to agriculture. We explore these interpretations in more detail below.

\subsection{Bi-square regression}

One of the major problems with ordinary least squares regression is its sensitivity to outlying observations as shown in figure 2 and as described by many authors (inter alia Unwin and Wrigley 1987, Wrigley 1983). Although a general trend is followed by most observations, there is one exception that unduly influences the position of the regression line.

Here the difficulty can be overcome by manual intervention, simply by excluding the outlying case from the regression analysis. However, this is only possible when the data can be visualised. If the regression model had three or more independent variables, outliers would have to be identified in Euclidean space of at least four dimensions. For this reason, some automated controlling for outliers needs to be adopted.

In its crudest form, this can be thought of as zero-weighting the offending observations. These can be identified in a two-stage process: 


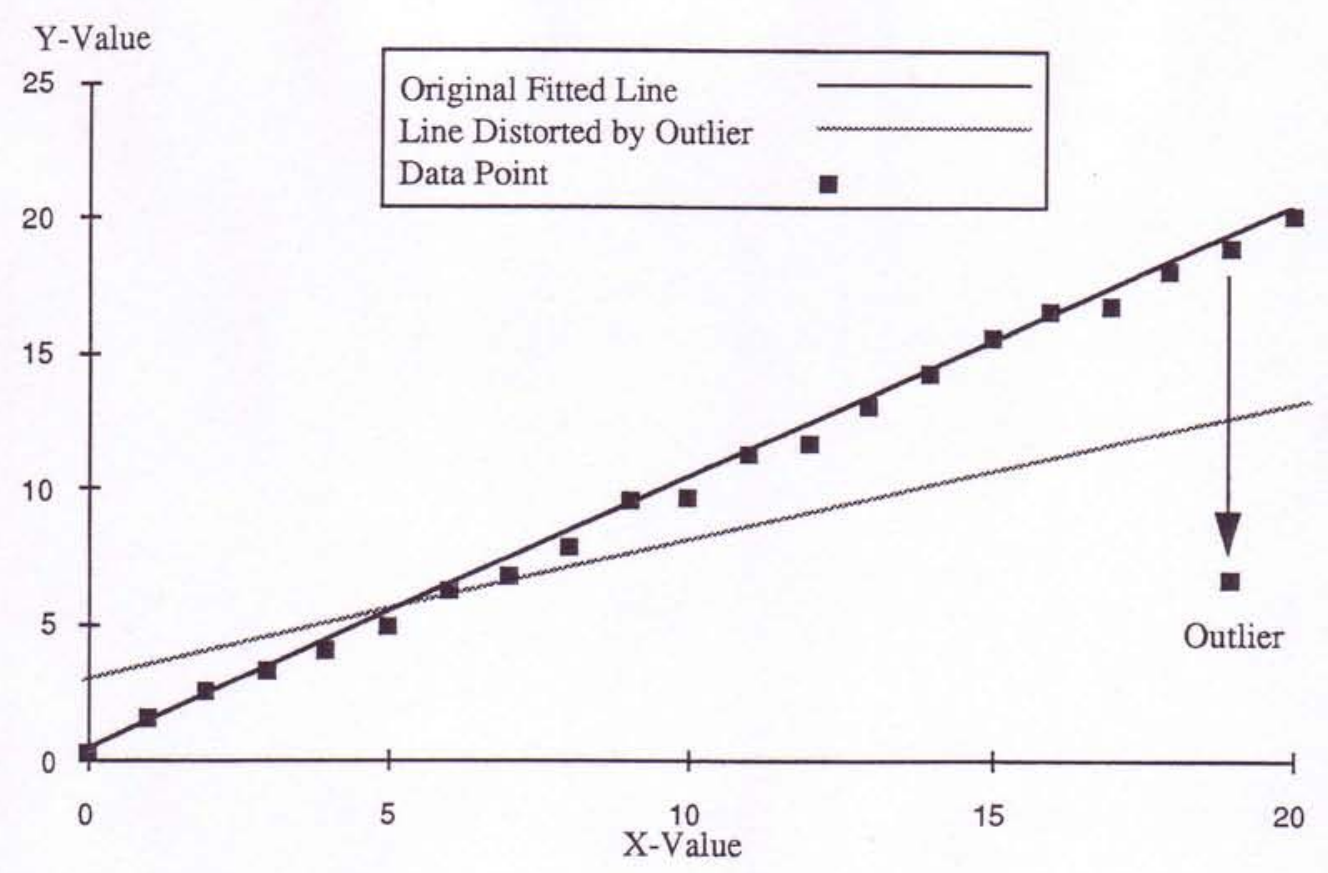

Figure 2. The effect of outliers on least squares regression.

1. Fit the model on all the data and compute the residuals.

2. Determine outliers by looking at the values of the residuals. Re-fit the model excluding these.

This seems a reasonable approach, but suffers from two main setbacks. Firstly, the threshold of residual size beyond which observations are deemed to be outliers is not specified. Secondly, the weighting makes a quantum leap from full weighting to absolute exclusion of the observation at this threshold. Thus, the weighting in the second stage can be thought of as a step function of $r$, the size of residual, and $k$, the threshold.

In the bi-weighted approach to outlier resistant regression, the cut-off is less drastic (Coleman et al. 1980). Weighting of the observations gradually falls off with increasing $r$, and finally becomes zero smoothly at the cut off point:

$$
\begin{array}{cc}
w(r)=\left(1-\left(\frac{r}{k}\right)^{2}\right)^{2} & \text { if } r \leqslant k \\
w(r)=0 & \text { otherwise }
\end{array}
$$

This is illustrated in figure 3. The question still arises as to the value of $k$ although a good experimental value for $k$ has been found to be five times the median absolute value of the residuals. Finally, although the methodology suggested so far suggests only two iterations (i.e., fit regression, re-weight and fit again), the bi-square method can be iterated several times until the estimates of the regression coefficients converge.

In this instance, the bi-square methodology is applied to the moving window regression analysis alongside the ordinary least squares methodology. Due to the relatively small size of the regression windows (49 pixels), the effect of an outlying observation could be quite prominent on the estimates of the regression parameters. It is intended that this method will identify any outlying pixels and reduce any distortion that these may cause. In the case when the results from the two analyses do not differ drastically, this will at least reassure us that the ordinary least squares methodology was not affected by outliers. 


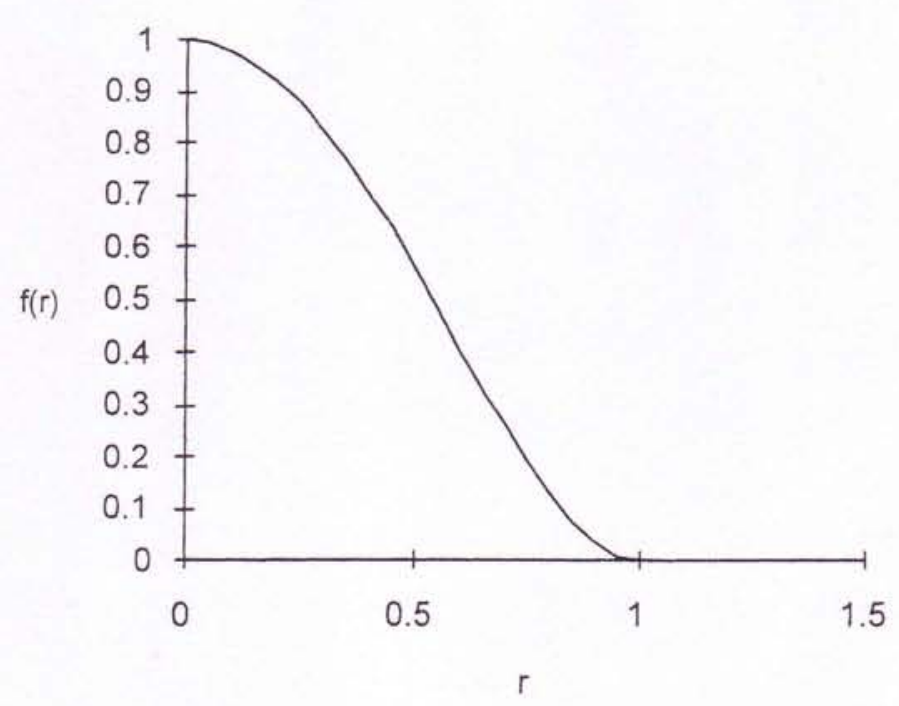

Figure 3. The weighting function in bi-square regression.

The result of calibrating the global bi-square regression model with standardised data is:

$$
\text { POPDEN }=-0.08-0.37 . E L-0.03 \text { WOOD }-0.07 \text { GRASS +0.03 PODS }
$$

which is similar to the OLS version although there are differences caused by the reduction in the influence of extreme values. It is not possible to report values of $t$ statistics for the parameter estimates because the standard errors reported in the bi-square calibration are unreliable because the weights are dependent on the observations and are therefore random variables. The $R$-squared value is unreliable for the same reason and should not be compared with that from the OLS regression.

\section{Localised results}

\subsection{Localised $O L S$ results}

To obtain localised results, a 7 by 7 window was placed over each pixel which provides 49 data points for the model calibration at each pixel location. This was the smallest square window with which we felt comfortable as a basis for analysis. The data for each set of 49 pixels are locally standardised prior to model calibration. As described above, the typical output of this technique for each of the four slope parameters in the model is a three-dimensional surface as shown in figure 4. A twodimensional representation is also shown as an alternative view of the data. Clearly there are variations in the parameter estimate over the region although it is not clear how important these variations might be. In an attempt to overcome this problem we have chosen to depict surfaces of the parameter estimates divided by their standard errors, $t$-statistic surfaces, which show more clearly the variations in results that can be obtained by using different parts of the data to calibrate the model. These $t$-statistic surfaces are shown in figures 5-8 for the parameters associated with the variables elevation, woodland, grassland, and podsols, respectively. The interpretation of these figures is that each point on the surface indicates the $t$-statistic associated with the parameter estimate that would be obtained if a 7 by 7 matrix of cells around that point constituted the sample from which the estimate was derived.

As an example, consider the interpretation of figure 5 which depicts the degree of non-stationarity for the parameter associated with elevation. The regions of upland depicted in white are those areas for which the local parameter estimate is significantly 

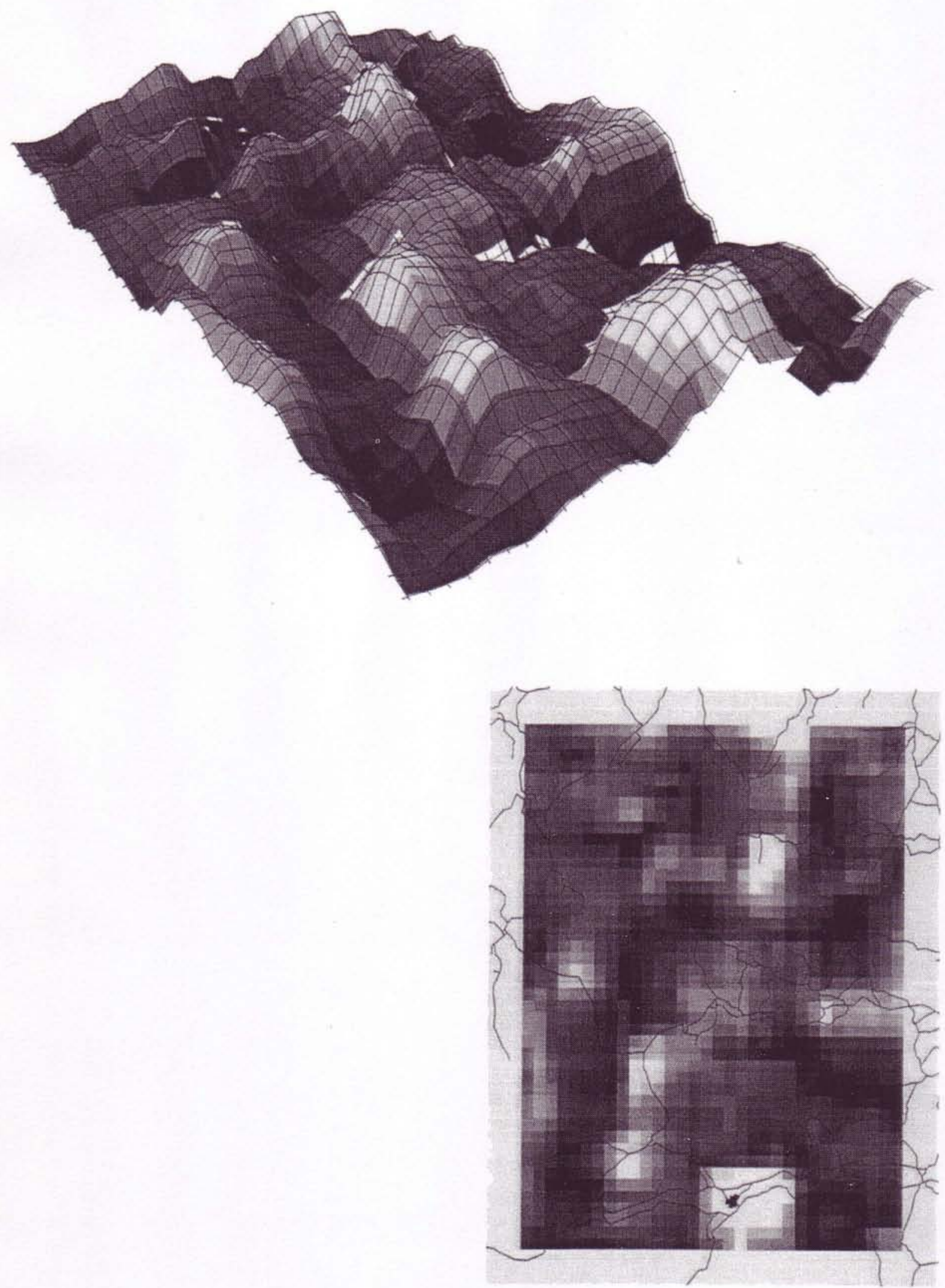

Figure 4. OLS model elevation parameter.

positive. The regions shaded in light-grey, the hill sides, are where the parameter estimate is not significantly different from zero, and the valley bottoms shaded in dark-grey depict areas where the parameter estimate is significantly negative. So whereas the global regression result suggests a significant negative relation between population density and elevation, there are a number of localised areas where 

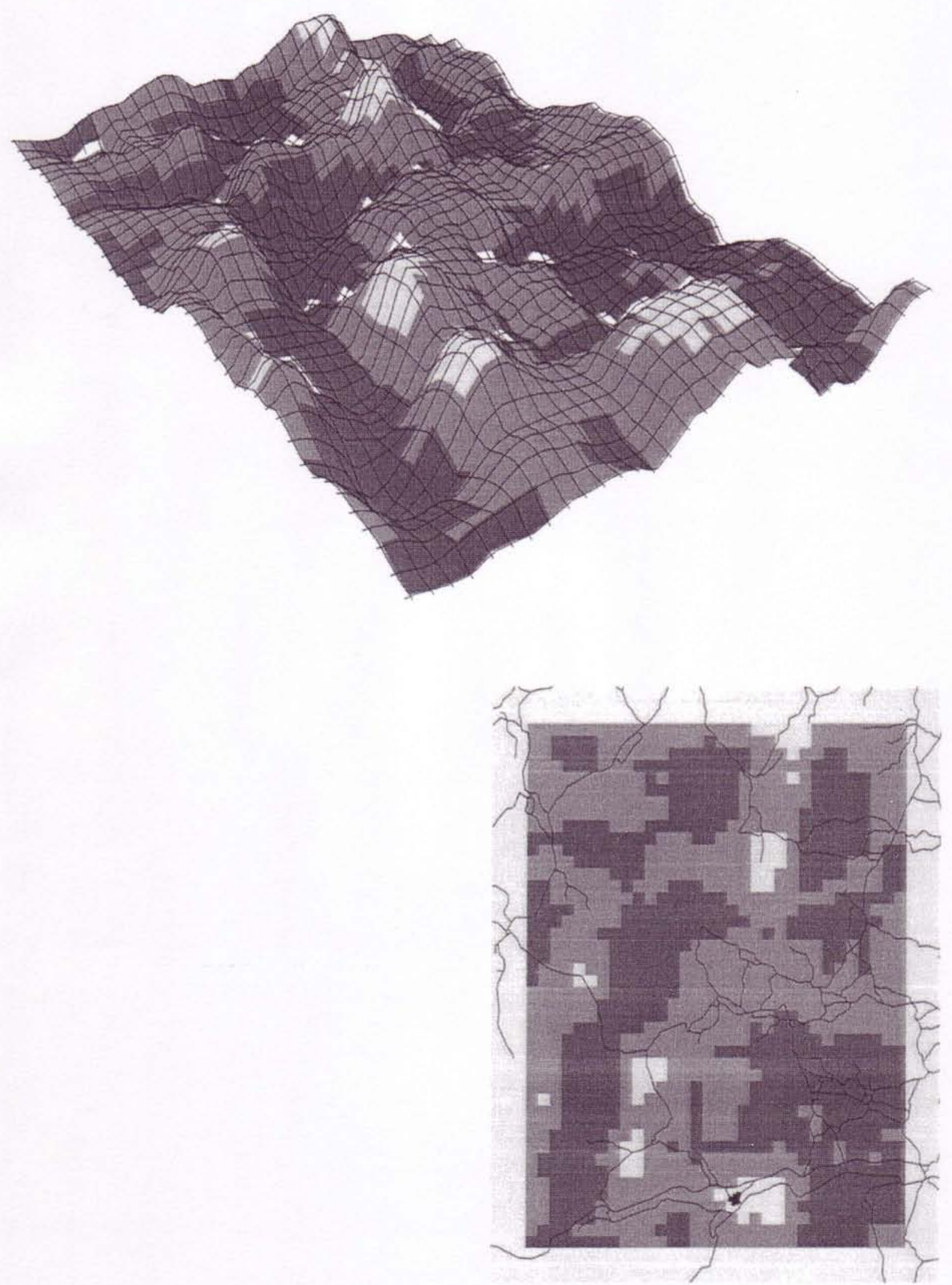

Figure 5. OLS model elevation $t$-statistic.

population density increases significantly as elevation increases. The two-dimensional map, onto which the local road pattern has been added, suggests that the areas where a significant positive relation between population density and elevation exists are close to roads which in this area tend to hug lower ground. This could suggest an aversion to very low-lying ground which may be difficult to cultivate or which may be prone to flooding. Whatever the explanation, the results are striking in their 

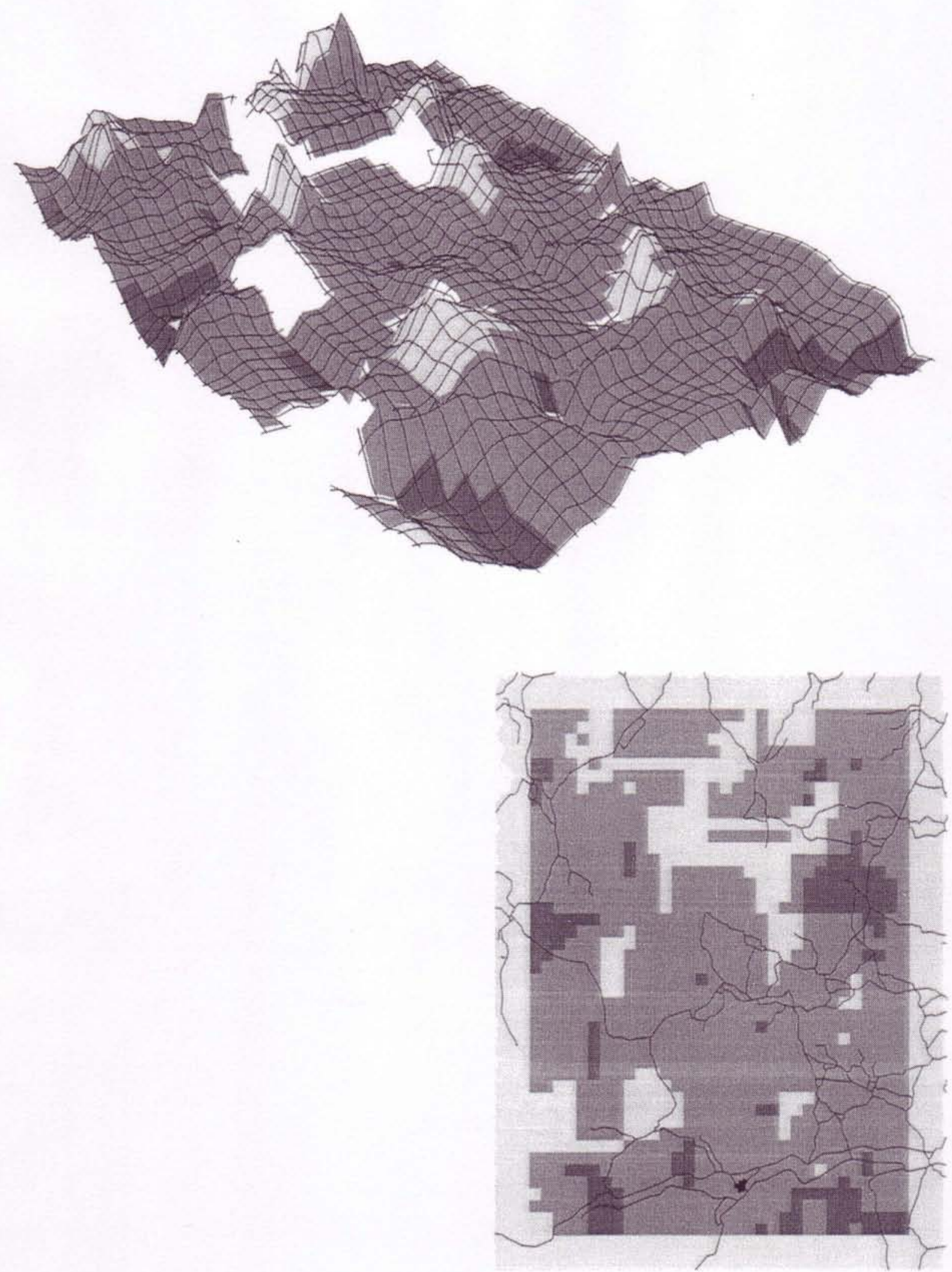

Figure 6. OLS model woodland $t$-statistic.

depiction of the extent of spatial non-stationarity which is completely hidden in the traditional modelling approach.

Because the vertical exaggeration is held constant throughout, figure 6 depicts a similar, although slightly less dramatic, picture for the woodland parameter. Use of the global data set would lead to the conclusion that there is a significant negative relation between population density and the amount of woodland although most of 

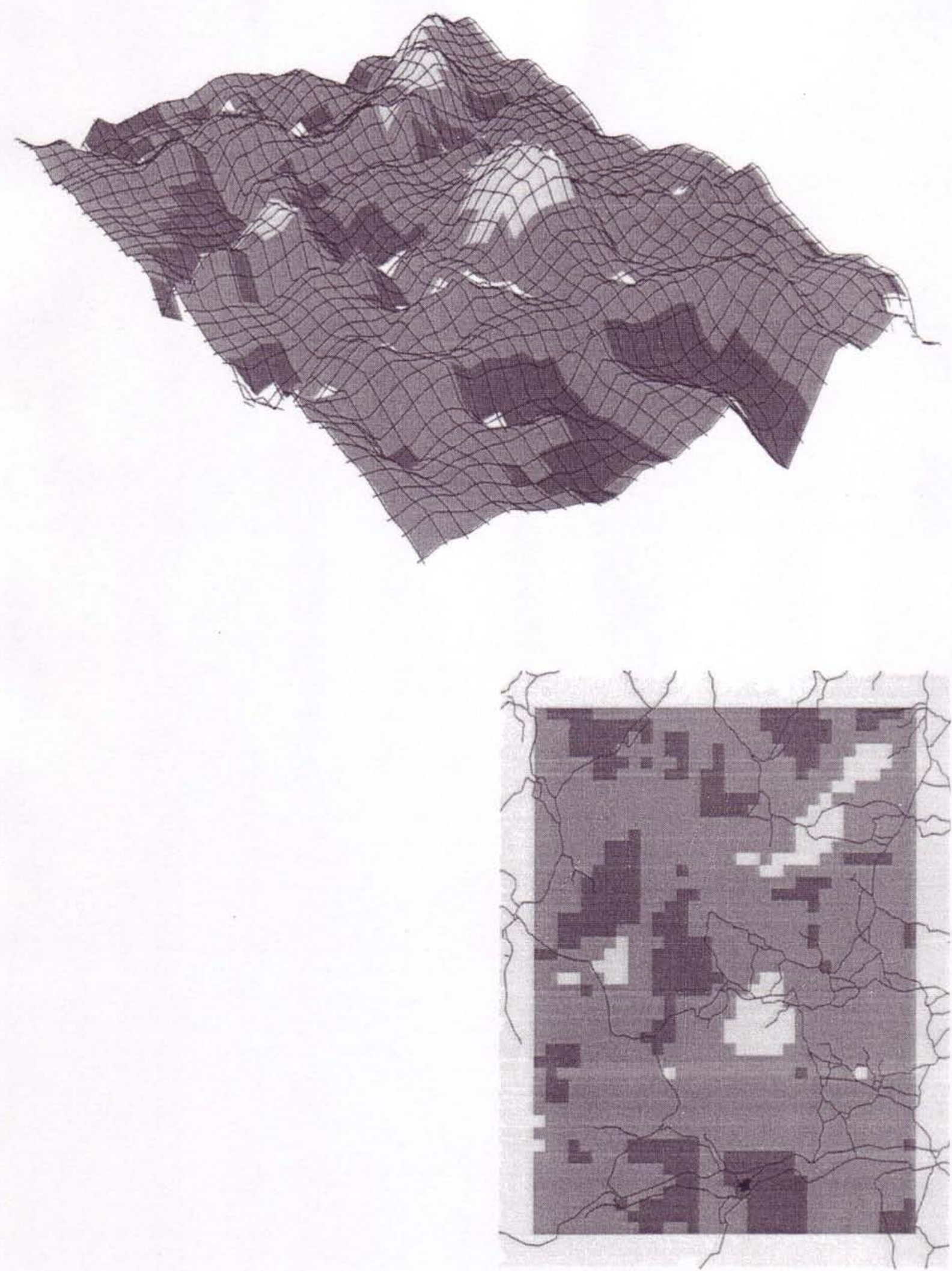

Figure 7. OLS model grassland $t$-statistic.

the local data sets would lead one to conclude that there is no significant relation. This apparent discrepancy is probably caused by the large number of degrees of freedom in the former although it should be noted that the global estimate is just significant at the 95 per cent confidence level. There are a number of local data sets which would lead to the conclusion that there is a significant positive relation. The pixels for which no results are reported on this surface are those for which the data 

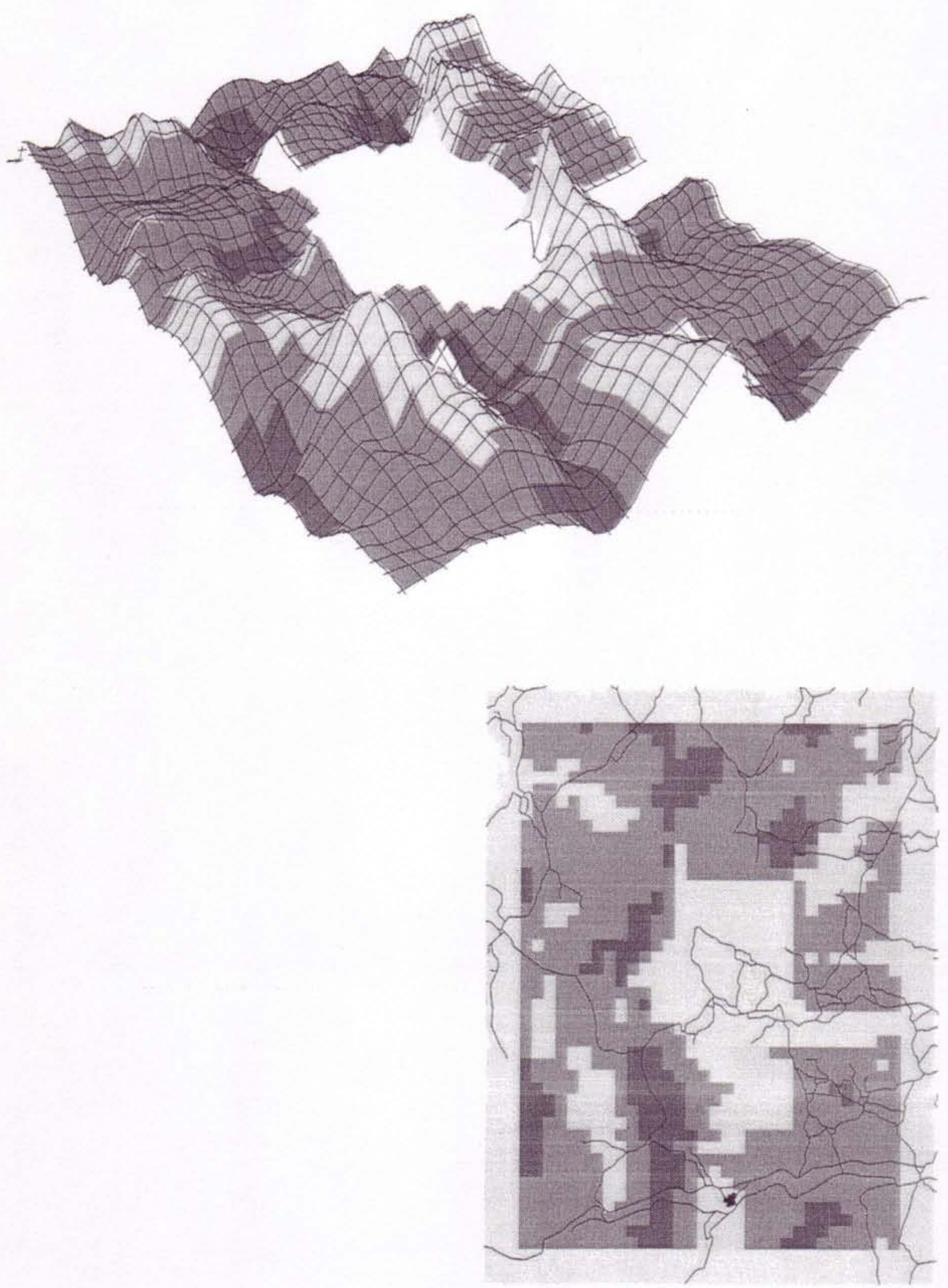

Figure 8. OLS model podsols $t$-statistic.

did not allow the local estimation of the parameter; this was because the variable was constant within the window.

The grassland estimates in figure 7 again suggest that one would reach the conclusion of no significant relation using many of the local data sets, in contrast to the globally significant negative relation. However, there are regions where the local data lead one to conclude the relation is significantly positive and others that 
lead one to conclude it is significantly negative. It needs more familiarity with the local geography to understand why the relations should show this degree of variation.

Figure 8 is dominated by a large area of missing values where the variable was zero (that is the soils were not podsolic) for all 49 pixels within the window. On the remainder of the surface there are substantial areas where the local data lead to the conclusion of a significant negative relation in contrast to the globally reported positive one.

The goodness-of-fit, measured by the $R$-squared statistic, of the local model is depicted in figure 9 which uses a continuous grey scale to show spatial variations in the degree to which the model fits the data. There are clearly identifiable hills and valleys in this surface. The model performance ranges from explaining only 1 per cent of the variance of the population density data in some parts of the region to explaining over 80 per cent in others. The globally fitted model explains 26 per cent of the variance. Again, the large spatial variation in goodness-of-fit suggests that spatial non-stationarity exists and that there are interesting fits to the data in some parts of the region, prompting questions regarding both the data and the model which need detailed local knowledge, perhaps from subsequent fieldwork, to answer. The surface therefore provides a good example of the use of this technique in encouraging a more detailed consideration of the data and the relations being examined than would happen in the conventional, global type of analysis currently the modus operandi of most research.

\subsection{Localised bi-square results}

Figures $10-13$ show the $t$-statistic surfaces produced by the bi-square calibration method. Given that this calibration method is designed to reduce the effects of outliers in the data, there will obviously be some differences between these surfaces and those produced by OLS. For reasons discussed earlier, any significance testing based on the bi-square technique is strictly exploratory. However, the differences are not particularly great and the bi-square surfaces depict a similar degree of spatial non-stationarity to the OLS ones and so will not be discussed further.

The $R$-squared surface shown in figure 14 again is similar to that derived from the OLS calibration method although the absolute values cannot be directly compared to those produced by OLS because of the difference in the two calibration methods.

The similarity of the two sets of results usefully demonstrates that the OLS regression is not being unduly biased by the presence of outliers. The methodology also shows how the comparison can be used to assess relative model performance because if the results of the localised calibration were quite different, then it may be possible to make statements about which model is better able to capture spatial variations in relations.

\subsection{A comparison of global, local OLS and local bi-square results}

A final set of results depicts the relative performances of the three estimation techniques in replicating the observed population density surface. In figure 15 , three sets of scatterplots show the relations between the observed data on the horizontal axis and the global predicted values (figure $15(a)$ ), the local OLS predicted values (figure 15(b)) and the local bi-square predicted values (figure 15(c)). It is clear, and only to be expected, that the local regressions outperform the global one in replicating the data since the former allow the mean of the predicted values to vary across the 

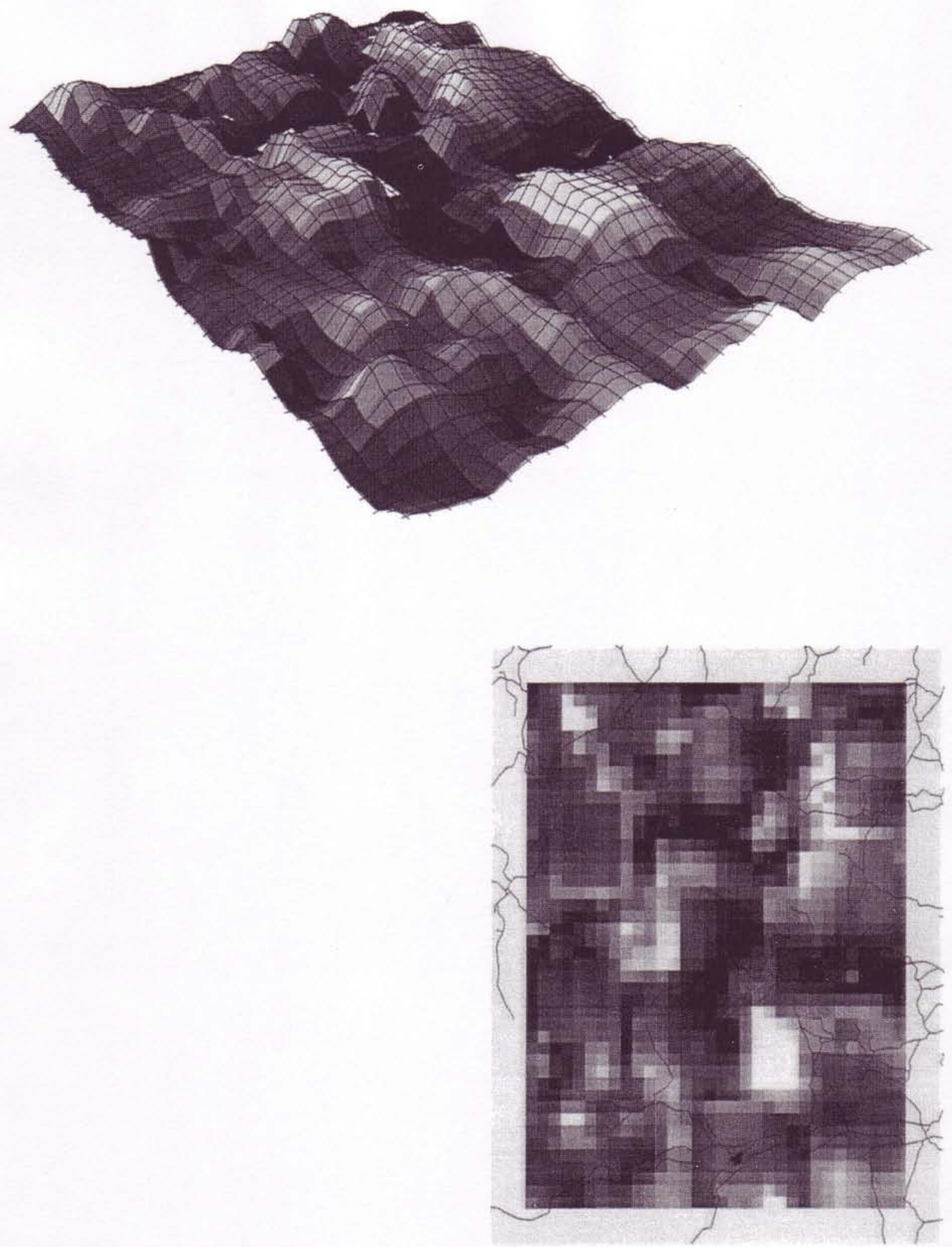

Figure 9. OLS model goodness-of-fit.

study area. Perhaps more interesting is the extent to which the global regression results fail to replicate the spatial pattern of the original data as shown in figure 16 . Population density in this part of NE Scotland shows a marked trend with highest values towards the south-east and lowest values towards the north-west. The two locally derived estimated surfaces both succeed in picking up this trend, because of the moving mean, but the global regression model performs poorly and almost misses the trend entirely. 

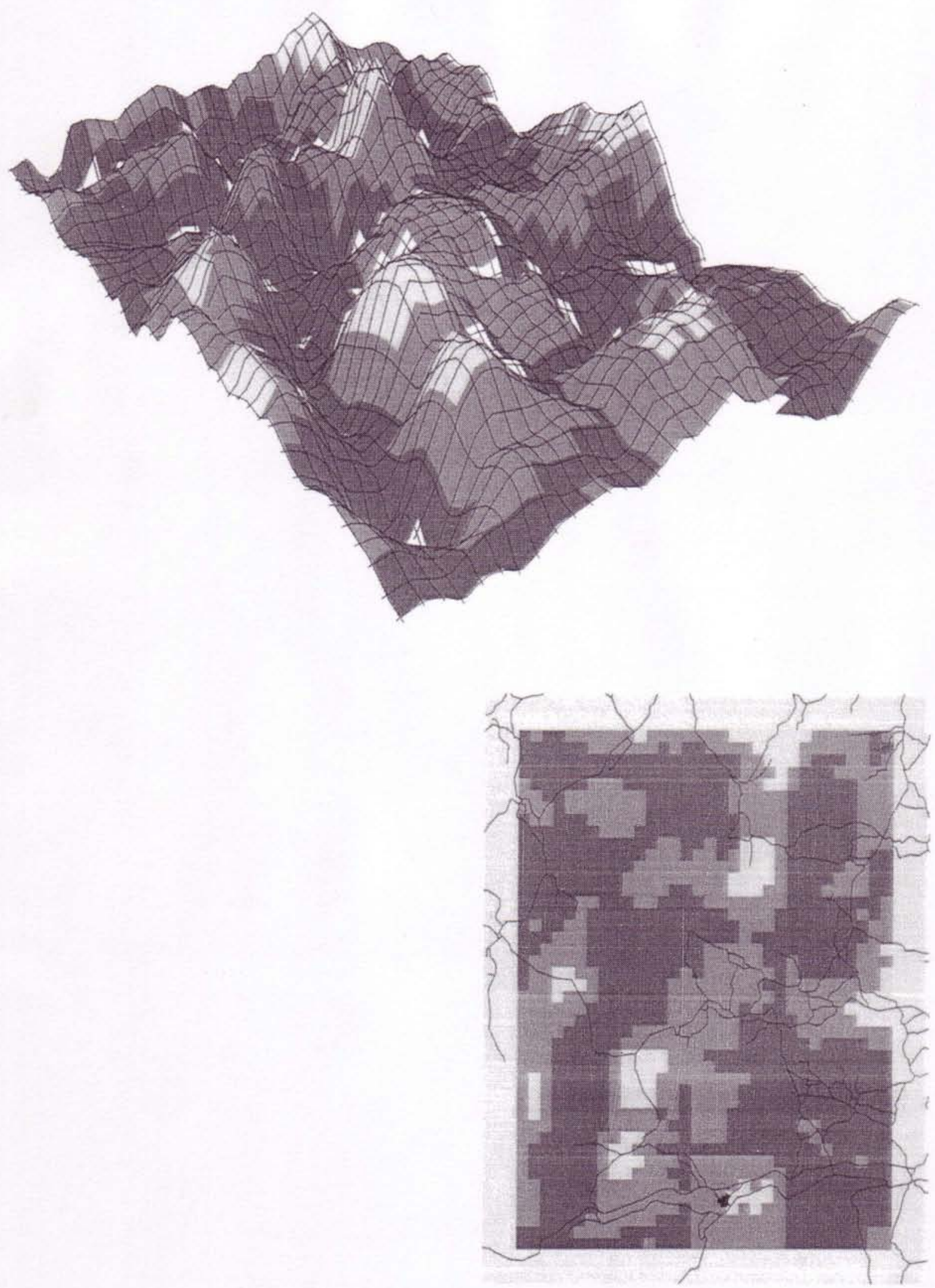

Figure 10. Bi-square model elevation $t$-statistic.

\section{Discussion}

This technique for the exploration and visualisation of spatial non-stationarity can be used on any spatial data set; however, the number of spatial units should be large enough to ensure that those units excluded from the analysis, due to the windowing, form a reasonably small percentage of the total. The technique is easier 

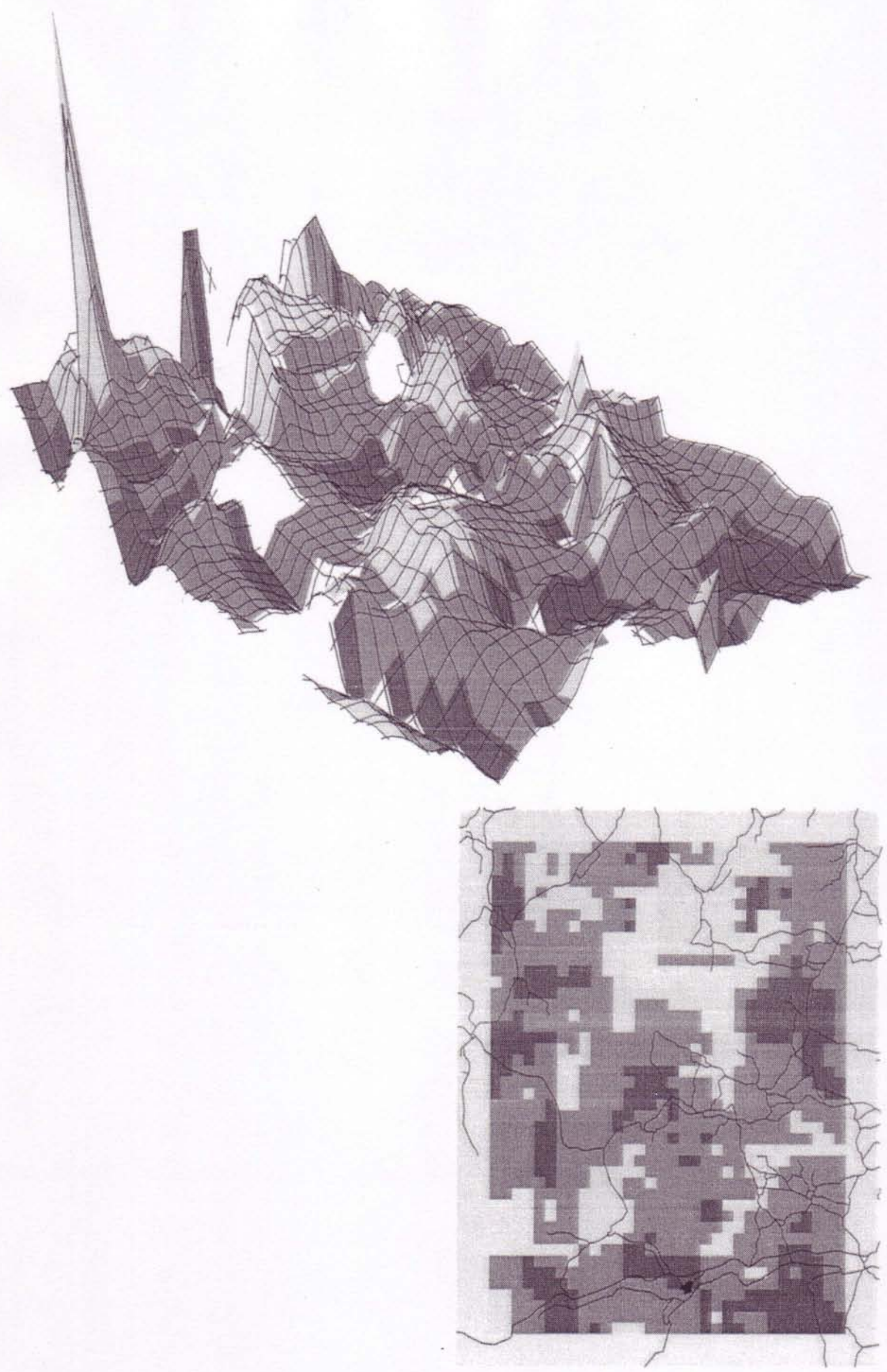

Figure 11. Bi-square model woodland $t$-statistic. 

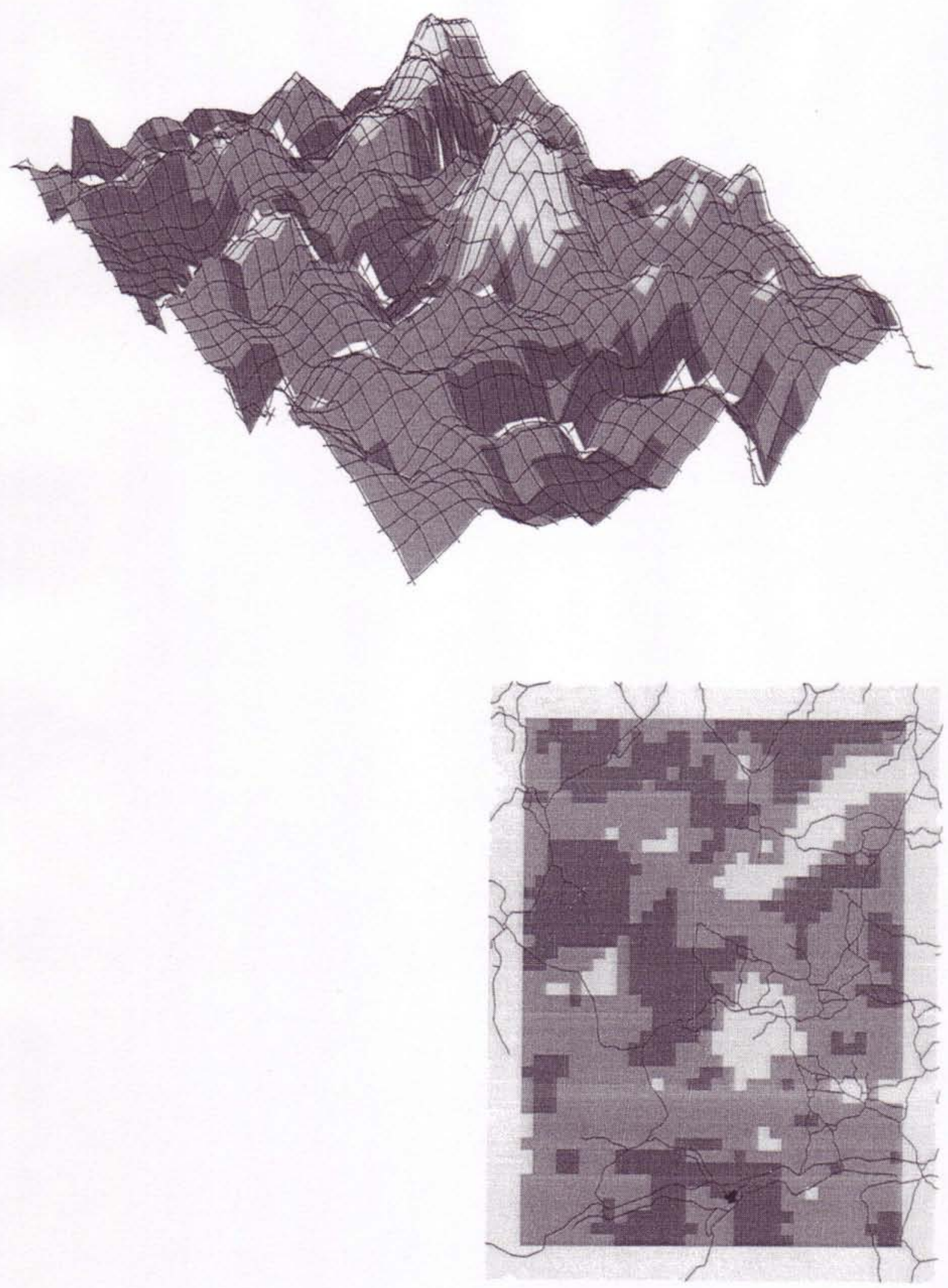

Figure 12. Bi-square model grassland $t$-statistic.

to apply to raster data due to the uniform nature of the recording units but there is no conceptual reason to restrict its use to this type of data. The technique could be applied to irregular polygons although some care would have to be taken in defining the window and this is currently under investigation.

Given that a key output from this technique is a set of three-dimensional surfaces depicting, inter alia, spatial variations in a set of parameter estimates, judging the 

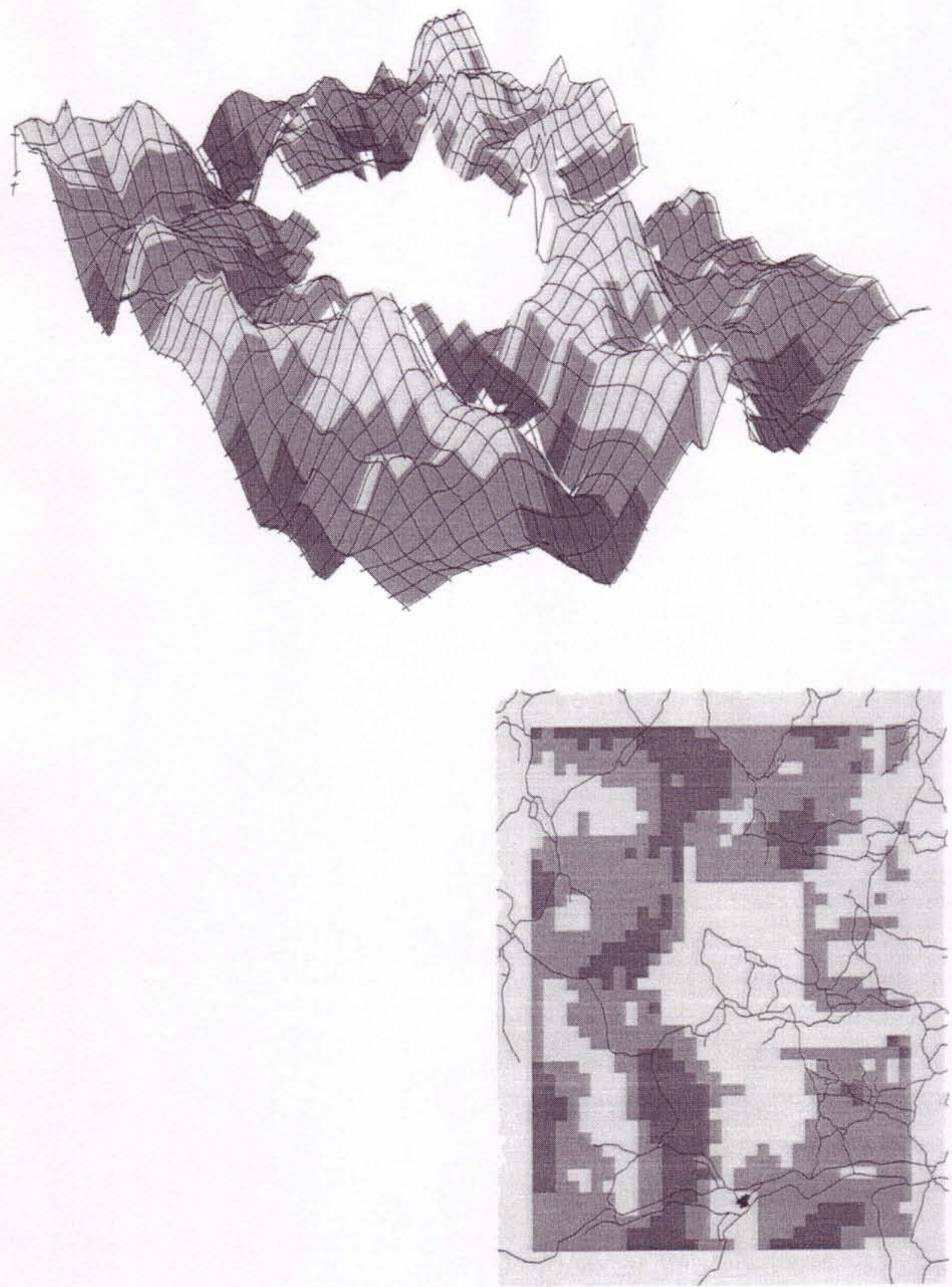

Figure 13. Bi-square model podsols $t$-statistic.

success of the technique is heavily visual and subjective. Consider, for example, a situation in which there was negligible spatial non-stationarity in a relation depicted by a parameter estimated through regression. The three-dimensional surface would then be a flat plain. Increasing deviations from this plain, as hills and valleys and other features evolve, indicate increasing degrees of spatial non-stationarity. Consequently, if the result from this technique is a relatively flat surface, then one 

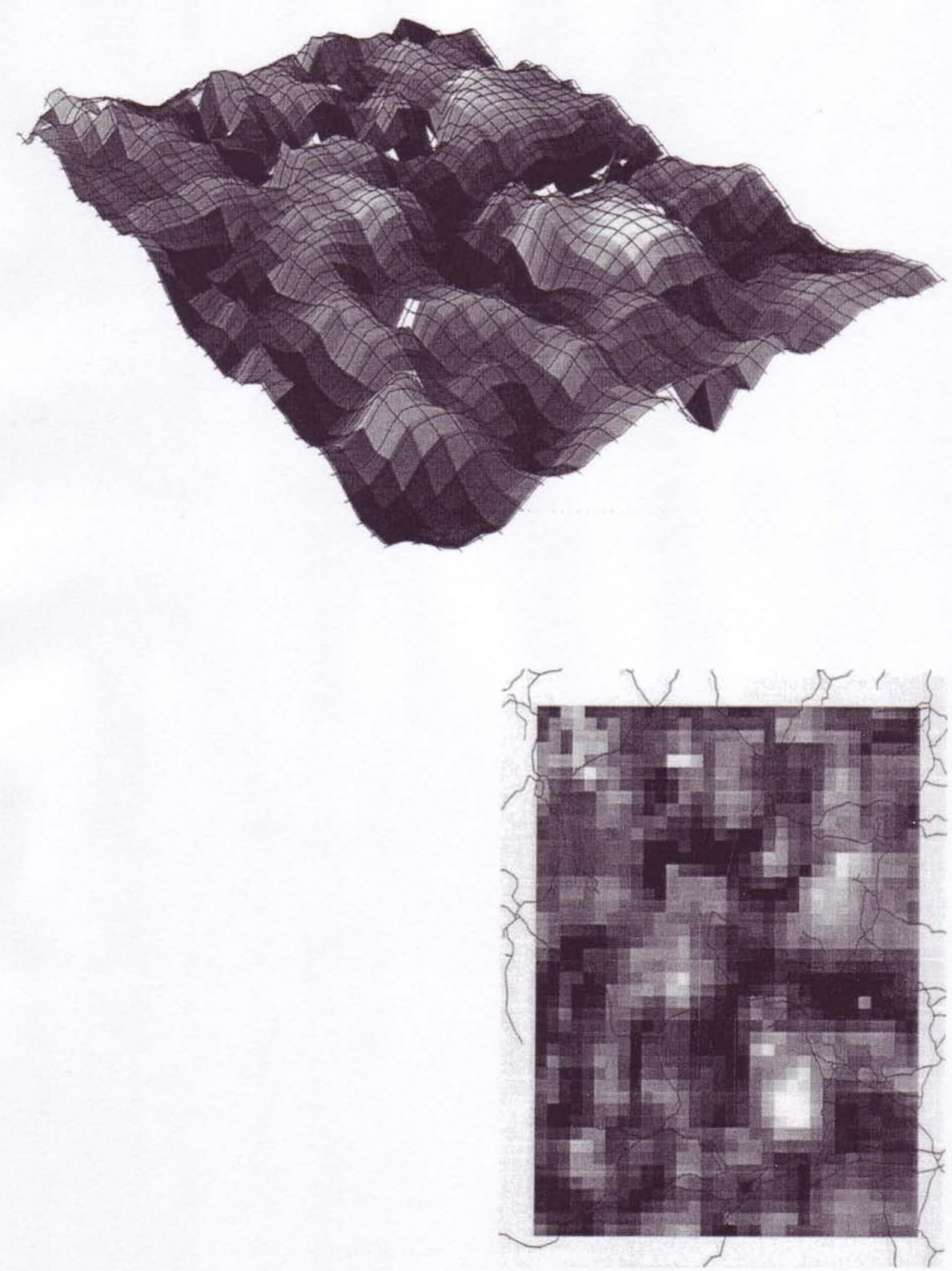

Figure 14. Bi-square model goodness-of-fit.

can infer that spatial non-stationarity is negligible which increases confidence in the global result as being a reasonable representation of a more general relation. However, if the output is a highly convoluted surface, then clearly the use of a global estimate is suspect.

The technique should be used for any modelling application where there is a suspicion of spatial non-stationarity. Although we have used a relatively simple 


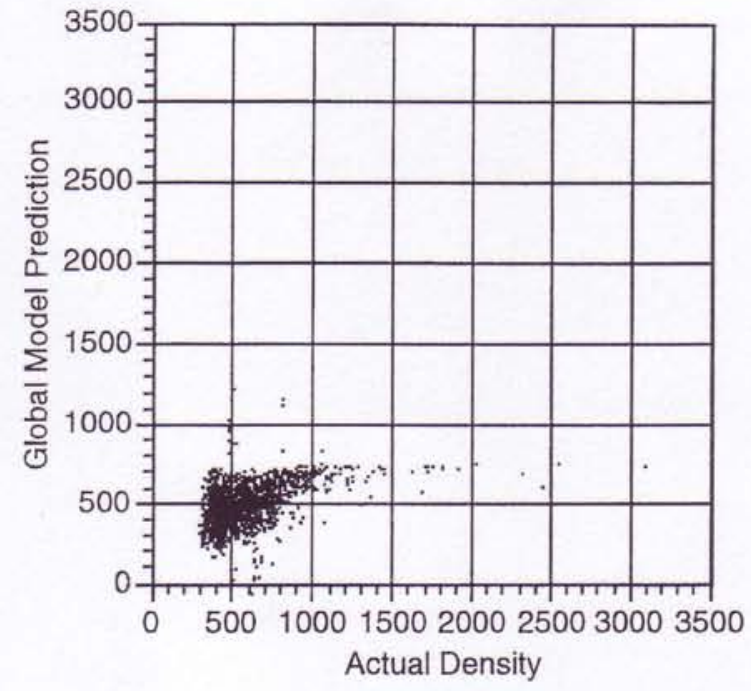

(a)

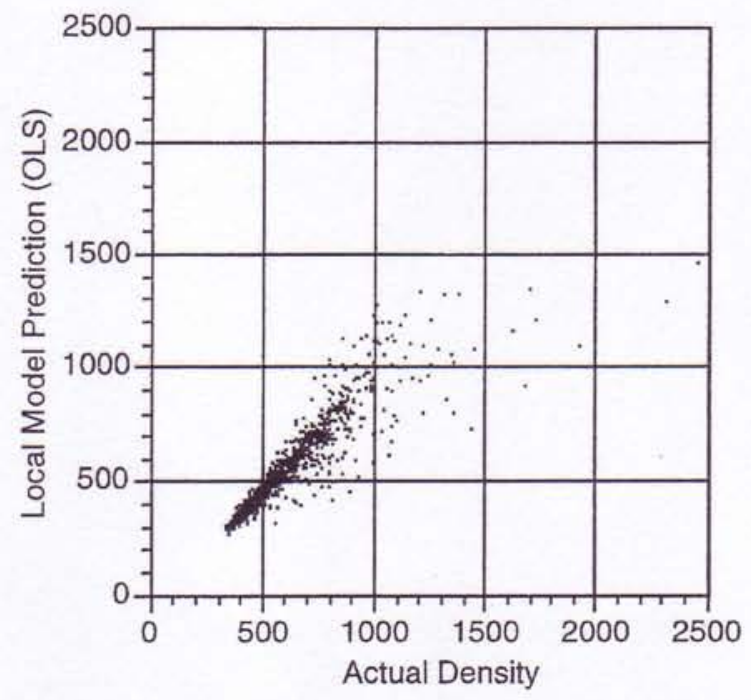

(b)

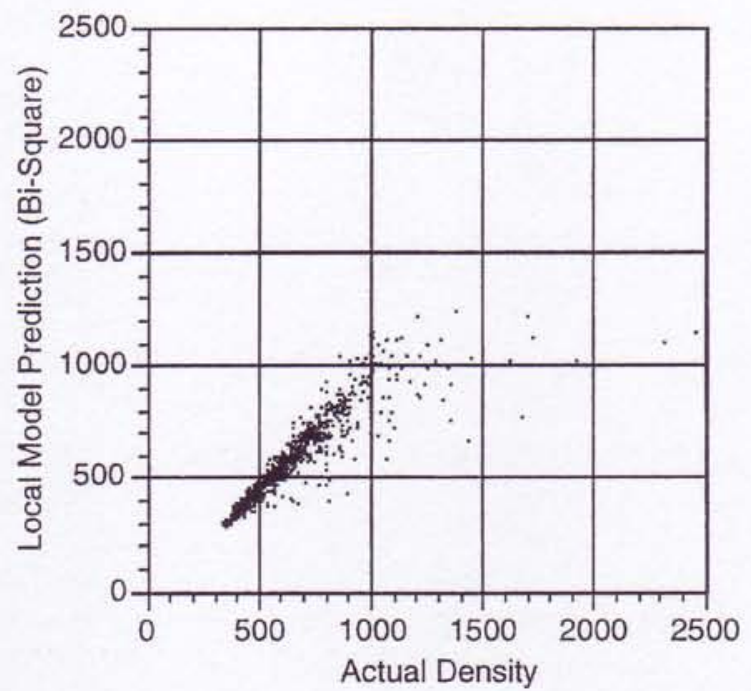

(c)

Figure 15. Comparison of fitted and actual density values. 


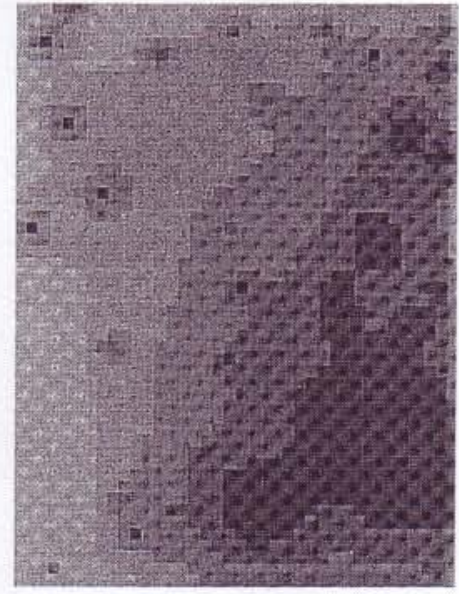

(a)

Population Density

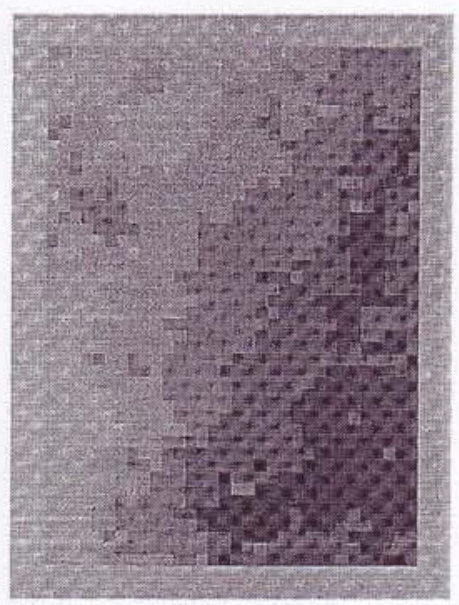

(c)

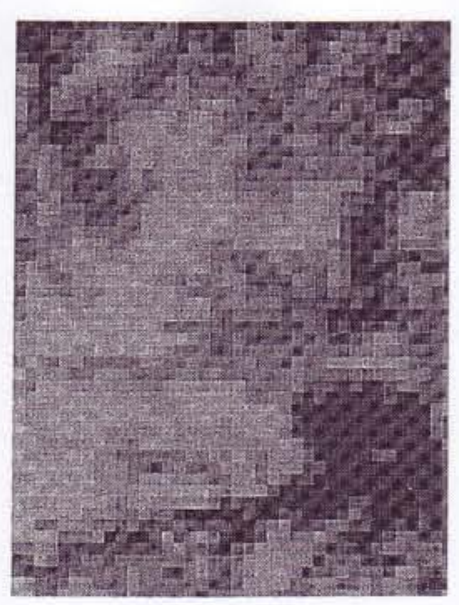

(b)

Global Prediction

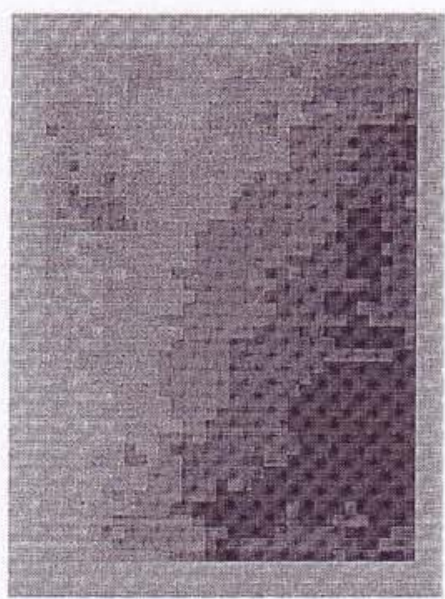

(d)

Local Predictions (Bisquare)

Local Predictions (OLS)

Figure 16. Spatial comparison of fitted and actual values.

linear model, with the justification that this is representative of the type of model used in many applications, the technique we describe can be used whenever it is useful to compare a global model with a series of local models. For instance, the model in question could just as easily be a non-parametric regression model (Hastie and Tibshirani 1990) which could be examined with a global data set and with many different subsets of the data to allow comparisons similar to those made above. We would argue that any globally-fitted model may miss important spatial variations in relations and that this technique will provide greater insights into the data being used for calibration as well as the nature of the relations being measured.

Given the emphasis on visualisation and the need to reference spatial co-ordinates, the technique should be applied through a GIS. It is not necessary to conduct the model calibrations within a GIS (and in the example described above the calibrations were undertaken outside a GIS) although the spatial referencing and graphics capabilities of a GIS make it almost a necessity in interpreting results. Hearnshaw and Unwin (1994), and particularly the chapters by Bracken (1994) and Gatrell (1994) therein, provide many other examples of the use of the visualisation capabilities of a GIS for exploratory spatial analysis. 


\section{Reservations}

We have the following reservations regarding the technique although we feel these are not sufficient to outweigh the benefits:

1. The main output is a set of three-dimensional surfaces which may be difficult to interpret. In some places, we use two-dimensional surfaces to depict information although the parameter results are best seen in three-dimensional to get a sense of the variations within the area. Of course, any three-dimensional plot is subject to the vertical exaggeration used in the construction of the surface.

2. Although all the original data are used in the construction of the threedimensional surfaces, not all the original cells of the region can be depicted because the outlying cells have to be 'stripped off' to provide consistency in the windowing. For example, if a 7 by 7 window is used on a raster image 500 by 500 , the dimensions of the resulting three-dimensional surface would be 494 by 494 . In most cases this will not present a problem unless the extreme margins of the region are of interest or the study area is small.

3. It is much easier to apply the technique to raster data where the cell size is uniform. Where cells are of unequal size, the definition of the window will be more difficult and the numbers of data points within the window may vary. However, neither of these problems should prohibit the use of the technique.

4. To avoid the problem of multiple hypothesis testing, the surfaces of $t$-values should be used primarily as exploratory tools and care should be taken on drawing inferences about the parameter estimates. The surfaces indicate those parts of the study area where particular conclusions would be reached regarding a relation if local data were used in the calibration of the model. The surfaces therefore prompt some interesting questions about the data and the relations being examined that would not otherwise get asked.

\section{Extensions}

In this research we have adopted a conservative posture by moving one step at a time. To this point we have explored non-stationarity in a very common model format, linear regression, which is available in many GISs. However, it is a model that has had several potential problems and given that one of the aims of this technique is to allow more detailed examination of any globally applied model, as well as to investigate spatial non-stationarity and data accuracy, an obvious extension would be to use the technique to evaluate modelling approaches designed explicitly to capture spatial effects. We intend to explore the capabilities of spatial lag and spatial error models in dealing with spatial non-stationarity. Would the parameter surfaces shown above be smoother if the estimates were drawn from either of these models capturing aspects of spatial dependency?

A further extensicn would be to apply the technique to a set of irregular polygons so that the optimal windowing technique for such a surface could be explored. In such an application it is likely that the window would be irregular and this could lead to a spatially adaptive windowing technique whereby windows of different magnitudes were allowed around each point in order to maximise some criterion, perhaps related to goodness-of-fit. It would also be interesting to examine the modifiable areal unit problem in the context of spatial non-stationarity: to what extent would the results change as the moving window changed in size? Would 
windows of different sizes identify non-stationarity at different scales? Presumably the surface will become smoother as window size increases and there may well be some optimal size of windowing that allows spatial trends in relations to be most clearly seen.

A final extension of the results would be to produce a more objective method of deciding when spatial non-stationarity is a serious problem. Clearly a threedimensional parameter surface that is flat is indicative of no spatial non-stationarity and increasing deviations from this plain, as hills and valleys and other features evolve, indicate increasing degrees of spatial non-stationarity. It would be useful to have an objective means of deciding whether the degree of spatial non-stationarity invalidated the use of a global model. Such a test may be possible based on explained variances and would be similar to the $F$-test used in ordinary regression.

\section{Summary}

The classical use of regression with spatial data is to calibrate a model, spatial or aspatial, to produce a global set of parameter estimates which are implicitly assumed to represent relations that are constant across the space from which the data are drawn. This paper provides a relatively simple technique for examining this assumption and in the example above the results indicate that the assumption is questionable. Given the results provided here, for example, how much faith could one place on any extrapolation of the global results beyond this particular set of polygons in north-east Scotland?

The methodology of depicting spatial non-stationarity visually is part of a general trend towards exploratory analysis particularly in the use of spatial data. Geographers have long been embarrassed by their data because of the difficulties arising from properties such as spatial dependency and spatial non-stationarity. These problems are increasingly seen as the outcomes of data which are rich in information and which provide the catalyst for increasingly imaginative types of analysis. It is our hope that this and subsequent studies will advance the understanding of the behaviour of models in the context of spatial data analysis.

\section{References}

Anselin, L., 1988, Spatial Econometrics: Methods and Models, (Dordrecht: Kluwer Academic). Aspinall, R. J., and VeItCH, N., 1993, Habitat mapping from satellite imagery and wildlife survey data using a Bayesian modelling procedure in a GIS. Photogrammetric Engineering and Remote Sensing, 59, 537-543.

BESAG, J. E., 1986, On the statistical analysis of dirty pictures. Journal of the Royal Statistical Society B, 48, 259-279.

BRACKEN, I., 1994, Towards improved visualization of socio-economic data. In Visualization in GIS edited by H. Hearnshaw and D.J. Unwin (Chichester: Wiley), pp. 76-84.

Coleman, D., Holland, P., Kaden, N., Klima, V., and Peters, S. C., 1980, A system of subroutines for iteratively re-weighted least-squares computations. ACM Transactions on Mathematical Software, 6, 327-336.

Cressie, N. A. C., 1991, Statistics for Spatial Data (New York: Wiley).

Flowerdew, R., and Green, M., 1994, Areal interpolation and types of data. In Spatial analysis and GIS edited by A.S. Fotheringham and P.A. Rogerson (London: Taylor \& Francis), pp. 121-145.

Fotheringham, A. S., and PITTS, T., 1995, Directional variation in distance-decay. Environment and Planning A, 27, 715-729.

Fotheringham, A. S., and Wong, D. W., 1991, The modifiable areal unit problem and multivariate analysis. Environment and Planning A, 23, 1025-1044. 
Gatrell, A., 1994, Density estimation and the visualization of point patterns. In Visualization in GIS edited by H. Hearnshaw and D.J. Unwin (Chichester: Wiley), pp. 65-78.

Getis, A., and ORD, J. K., 1991, The analysis of spatial association by use of distance statistics. Geographical Analysis, 23, 189-206.

Hastie, T. J., and Tibshirani, R. J., 1990, Generalized Additive Models (London: Chapman \& Hall).

Hearnshaw, H., and UnwIN, D. J., 1994, Visualization in GIS (Chichester: Wiley).

JONES, J. P. III, and CASETTI, E., 1992, Applications of the Expansion Method (London: Routledge).

MARTIN, D., 1989, Mapping population data from zone centroid locations. Transactions of the Institute of British Geographers, 14, 90-97.

UNWIN, D. J., and WrIGLEY, N., 1987, Control point distribution in trend surface modelling revisited: an application of the concept of leverage. Transactions of the Institute of British Geographers, 12, 147-160.

Wrigley, N., 1983, Quantitative methods: on data and diagnostics. Progress in Human Geography, 7, 567-577. 